\title{
Factor V Leiden related Budd-Chiari syndrome
}

\author{
P Deltenre, M-H Denninger, S Hillaire, M-C Guillin, N Casadevall, J Brière, S Erlinger, \\ D-C Valla
}

\begin{abstract}
Background-The role of factor $V$ Leiden as a cause of Budd-Chiari syndrome has only recently been described.

Aims-To assess the specific features of factor V Leiden related Budd-Chiari syndrome.

Patients-Sixty three consecutive patients with hepatic vein or terminal inferior vena cava thrombosis.

Methods-Standardised chart review.

Results-Factor V Leiden was found in $\mathbf{2 0}$ patients $(31 \%(95 \%$ CI $20-43))$. In the subgroup of patients with, compared with the subgroup without, factor V Leiden, a combination of prothrombotic states was more common (70\% (95\% CI 50-90) $v$ 14\% (95\% CI 3-24)); inferior vena cava thrombosis was more frequent $(40 \%$ (95\% CI 19-61) $v$ 7\% (95\% CI 0-14)); and distribution of initial alanine aminotransferase values was bimodal (almost normal or extremely increased) versus unimodal $(p=0.003)$. Factor $V$ Leiden accounted for four of five cases of massive ischaemic necrosis (transaminases $>50$-fold the upper limit of normal values) $(p=0.014)$, and also for all three cases developing during pregnancy. Patients with and without factor $V$ Leiden did not differ with regard to mortality, portosytemic shunting, or listing for liver transplantation. Hepatocellular carcinoma developed in two patients; both had factor V Leiden and indolent obstruction of the inferior vena cava.
\end{abstract}

Conclusions-In patients with BuddChiari syndrome, factor $V$ Leiden (a) is common; (b) precipitates thrombosis mostly when combined with another risk factor; (c) is associated with one of two contrasting clinical pictures: indolent thrombosis-particularly of the inferior vena cava-or massive ischaemic necrosis; and (d) is a major cofactor of Budd-Chiari syndrome developing during pregnancy.

(Gut 2001;48:264-268)

d'Hématologie,

Hôpital Raymond

Poincaré, Garches,

France

N Casadevall

Correspondence to: Dr D-C Valla, Service d'Hépatologie, Hôpital Beaujon, 100, Boulevard du Général Leclerc, 92118

Clichy, France.

dominique.valla@,

bjn.ap-hop-paris.fr

Accepted for publication 18 July 2000 outflow, leading to sinusoidal congestion ischaemic injury to liver cells, and portal hypertension. The main mechanism of obstruction is thrombosis of hepatic veins or terminal portion of the inferior vena cava.
Thrombosis at these sites usually occurs in association with various prothrombotic disorders, including primary myeloproliferative disorders, antiphospholipid syndrome, paroxysmal nocturnal haemoglobinuria, inherited deficiency in natural coagulation inhibitors, and miscellaneous prothrombotic disorders. ${ }^{1-8}$ Pregnancy and oral contraceptive use are regarded as precipitating cofactors. ${ }^{129}$ However, in $10-30 \%$ of patients, a prothrombotic state cannot be identified.

Recently, the so called factor V Leiden has been recognised as a common prothrombotic disorder. Factor $\mathrm{V}$ Leiden results from the G1691A factor $V$ gene transition. This anomaly induces resistance of activated factor $\mathrm{V}$ to degradation by activated protein $\mathrm{C} .{ }^{10} \mathrm{Fac}-$ tor V Leiden is found in about $25 \%$ of patients with deep vein thrombosis. ${ }^{10}$ Anecdotal cases of Budd-Chiari syndrome associated with factor $\mathrm{V}$ Leiden have been reported. ${ }^{11-15}$ In a recent survey, seven of 30 cases of Budd-Chiari syndrome were found to be associated with factor V Leiden. ${ }^{16}$ However, the respective roles of factor $\mathrm{V}$ Leiden and myeloproliferative disorders could not be accurately assessed because occult forms of the latter-a common cause of Budd-Chiari syndrome-had not been evaluated.

The aim of this study was to ascertain the role of factor $\mathrm{V}$ Leiden in hepatic vein or inferior vena cava thrombosis and to examine the characteristics of factor $\mathrm{V}$ Leiden related Budd-Chiari syndrome.

\section{Patients and methods}

We studied 63 consecutive patients with BuddChiari syndrome due to thrombosis of the hepatic veins or inferior vena cava, seen between 1994 and 1999. The following prothrombotic disorders were looked for in every patient using previously reported methods and criteria: classical primary myeloproliferative disease fulfilling established criteria regarding platelet count, total red cell mass, endogenous erythroid colonies, and bone marrow biopsy $^{17}{ }^{18}$; formes frustes of primary myeloproliferative disorders, not fulfilling classical criteria but with endogenous erythroid colonies ${ }^{19}$; paroxysmal nocturnal haemoglobinuria ${ }^{20}$; antithrombin activity, protein $\mathrm{C}$ activity, and protein $\mathrm{S}$ antigen deficiencies using commercial kits (Diagnostica Stago, Asnieres, France) and only considered as primary (that is, not related to impaired liver function) when other inhibitors and clotting factors were normal; anticardiolipin antibodies (Biogenic, Montpel-
Abbreviations used in this paper: ALT, alanine aminotransferase. 
Table 1 Prothrombotic states and risk factors for thrombosis in 63 patients with Budd-Chiari syndrome

\begin{tabular}{lll}
\hline Prothrombotic state or risk factor & No tested & No positive \\
\hline Primary myeloproliferative disorders & 61 & 31 \\
$\quad$ Forme fruste & & 15 \\
Overt form & 63 & 16 \\
G1691A factor V gene transition (factor V Leiden) & 63 & 20 \\
Anticardiolipin antibodies & 21 & 4 \\
Isolated protein C deficiency & 47 & 3 \\
G20210A prothrombin gene transition & 63 & 1 \\
Paroxysmal nocturnal haemoglobinuria & 30 & 2 \\
Isolated protein S deficiency & 21 & 1 \\
Inherited plasminogen deficiency & 47 & 0 \\
Antithrombin deficiency & & 3 \\
Pregnancy & & 6
\end{tabular}

^In only 21 patients without decreased levels of clotting factors could primary natural coagulation inhibitor or plasminogen deficiencies be reliably investigated. However, despite decreased coagulation factors, normal levels of antithrombin and protein $\mathrm{S}$ were found in 26 and nine patients, respectively. lier, France); lupus anticoagulant ${ }^{21} ; \mathrm{G} 1691 \mathrm{~A}$ factor $\mathrm{V}$ gene mutation ${ }^{22}$ or resistance to activated protein $\mathrm{C}^{23}$; and, in patients seen after 1996, G20210A prothrombin gene mutation. ${ }^{24}$ Assessment of coagulation inhibitors and clotting factor deficiencies was performed at least one month after an episode of thrombosis. Some of the patients included in the present study had also been included in a recently reported study on the concurrence of prothrombotic states in patients with hepatic vein thrombosis. $^{25}$

Hepatic veins, portal vein, and/or inferior vena cava were studied using ultrasonography, magnetic resonance imaging, computed tomography, or angiography, as appropriate. Tumorous hepatic vein invasion or compression was excluded as appropriate. Acute ischaemic necrosis was considered when serum alanine aminotransferase (ALT) activity was greater than five times the upper limit of the normal range. Massive ischaemic necrosis was considered when there were signs of liver failure in association with serum ALT activity exceeding 50 times the upper limit of normal.

Comparison of the characteristics of patients with and without factor V Leiden mutation was performed using the Student's $t$ test for qualitative data and the $\chi$ test with Yates' correction for quantitative data.

\section{Results}

Prothrombotic disorders, risk factors as well as associated, possibly causal, conditions are outlined in table 1. Apart from factor V Leiden, all prothrombotic disorders could not be reliably tested for various reasons: associated decrease in the level of clotting factors suggesting liver insufficiency with secondary deficiencies in coagulation inhibitors in 39 patients (11 patients with and 30 patients without factor $\mathrm{V}$ Leiden); unable to assess myeloproliferative disorders in two critically ill patients with factor V Leiden; and mutation G20210A of the factor II gene not yet recognised at the time the patients were seen (five patients with and nine patients without factor V Leiden).

Resistance to activated protein $\mathrm{C}$ was found in 20 patients $(31 \%$ (95\% confidence interval (CI) 20-43)). Factor V Leiden mutation was tested in 19 of 20 patients with resistance to activated protein $\mathrm{C}$ and in 38 of 43 patients without resistance to activated protein C. There was $100 \%$ concordance between the presence of factor $\mathrm{V}$ Leiden mutation and resistance to activated protein C. All patients with factor V Leiden were heterozygous for the mutation The other identified prothrombotic disorders are presented in table 1. As shown in table 2, combinations of various prothrombotic states or risk factors for thrombosis were found in several patients. In six patients, no prothrombotic state was identified.

Patient characteristics according to the presence or absence of factor $\mathrm{V}$ Leiden are presented in table 2. Age, sex ratio, prevalence of oral contraceptive use or heavy smoking, and prevalence of ascites did not significantly differ among patients with and without the mutation. Similarly, serum albumin and serum bilirubin levels as well as prothrombin time were similar in the two subgroups. Apart from factor V Leiden, the prevalence of prothrombotic states or other thrombophilic factors was similar in both subgroups. Thus a combination of two prothrombotic states was more commonly found in patients with factor $\mathrm{V}$ Leiden than in patients without this anomaly $(70 \%$ (95\% CI 50-90) v 14\% (95\% CI 3-24); p<0.005). However, the type and prevalence of associated

Table 2 Characteristics of patients with or without factor $V$ Leiden mutation

\begin{tabular}{|c|c|c|c|}
\hline & $\begin{array}{l}\text { With factor } \\
(n=20)\end{array}$ & $\begin{array}{l}\text { Without factor } \\
(n=43)\end{array}$ & $p$ \\
\hline Mean (SD) age (y) & $37(11)$ & $35(12)$ & NS \\
\hline Sex ratio $(M / F)$ & $5 / 15$ & $10 / 33$ & NS \\
\hline OC users/child bearing aged women & $4 / 15$ & $19 / 32$ & NS \\
\hline Heavy smoker $(\mathrm{n} \%)$ & $6(30)$ & $14(43)$ & NS \\
\hline Prothrombotic states (total No found/total No tested) & $38 / 94$ & $44 / 239$ & 0.00001 \\
\hline Prothrombotic states, excluding factor V Leiden (total No found/total No tested) & $18 / 74$ & $44 / 239$ & NS \\
\hline At least 2 prothrombotic states $(\mathrm{n}(\%))$ & $14(70)$ & $6(14)$ & $<0.005$ \\
\hline Isolated antithrombin deficiency ${ }^{\star}$ & $0 / 15$ & $0 / 32$ & NS \\
\hline Isolated protein $\mathrm{C}$ deficiency ${ }^{\star}$ & $1 / 9$ & $3 / 13$ & NS \\
\hline Isolated protein $\mathrm{S}$ deficiency ${ }^{\star}$ & $1 / 13$ & $1 / 17$ & NS \\
\hline G20210A prothrombin gene transition (No found/No tested) ${ }^{\star \star}$ & $2 / 15$ & $1 / 32$ & NS \\
\hline Primary myeloproliferative disorder (No found/No tested) & $6 / 18$ & $26 / 43$ & NS \\
\hline Acute ischaemic necrosis (ALT > 5 ULN) (n (\%)) & $6(30)$ & $22(51)$ & NS \\
\hline Massive ischaemic necrosis (ALT > 50 ULN) (n (\%)) & $5(25)$ & $2(4.6)$ & 0.017 \\
\hline Ascites (n (\%)) & $17(85)$ & $35(81)$ & NS \\
\hline Inferior vena cava thrombosis (n (\%)) & $8(40)$ & $3(7)$ & 0.0013 \\
\hline Portal vein thrombosis (n (\%)) & $2(10)$ & $11(25)$ & NS \\
\hline
\end{tabular}

^Number with isolated deficiency/number without decreased coagulation factor levels

${ }^{\star \star}$ Only patients seen after 1996 could be tested. OC, oral contraceptives; ULN, upper limit of normal. 


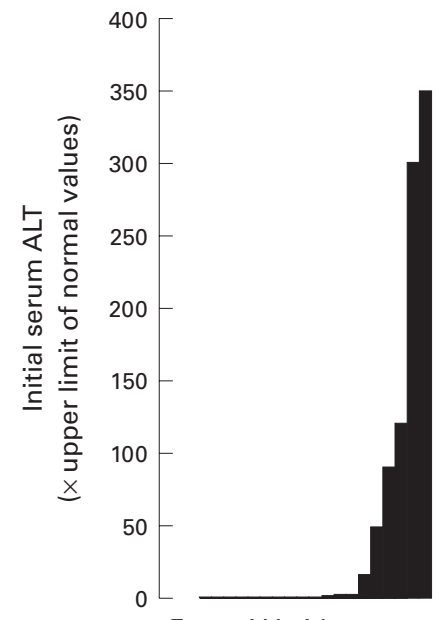

Factor V Leiden present

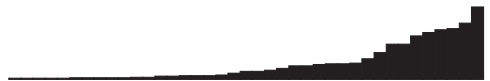

Factor V Leiden absent
Figure 1 Serum alanine aminotransferase (ALT) activity at the time of presentation in 63 patients with Budd-Chiari syndrome due to hepatic vein or inferior vena cava thrombosis according to the presence or absence of factor V Leiden mutation (each bar represents an individual value).

prothrombotic disorders in the subgroup of patients with factor $V$ Leiden were not different from those in patients without factor $\mathrm{V}$ Leiden.

As depicted in fig 1, at the time of presentation, absence of ongoing ischaemic injurywhich was assumed because of normal serum transaminase activity - was more common in patients with factor V Leiden (35\% (95\% CI 14-56)) than in those without factor $\mathrm{V}$ Leiden (11\% (95\% CI 2-21)). However, data presented in fig 1 show that, in patients with factor $\mathrm{V}$ Leiden and ongoing ischaemic injury (transaminases above five times the upper limit of normal), the magnitude of the elevation in ALT was much more marked than in patients without factor V Leiden. Indeed, factor V Leiden accounted for four of the five cases of massive liver injury (ALT >50-fold the upper limit of normal) encountered in this cohort $(p=0.014)$. In these four cases (table 3, patient Nos 17-20), an unusual acute condition was associated and obviously triggered development of massive ischaemic injury: eclampsia in a patient with an associated G20210A factor II gene mutation; fetal death in another pregnant patient with an associated G20210A factor II gene mutation; high dose factor VIII administration for pregnancy related factor VIII inhibitor; and catastrophic antiphospholipid syndrome triggered by profound hypothyroidism with shock in a young oral contraceptive user with Down's syndrome. No such unusual conditions were encountered in patients without factor V Leiden or in those without massive ischaemic injury. It is noteworthy that the three cases of pregnancy related Budd-Chiari syndrome belonged to the group of patients with factor V Leiden and massive ischaemic injury.

Inferior vena cava thrombosis was documented in eight of 20 patients with factor $\mathrm{V}$ Leiden $(40 \%$ (95\% CI 19-61)) but only in three of 43 patients without factor V Leiden $(7 \% \quad(95 \%$ CI $0-14)) \quad(p=0.0013)$. Hence factor $\mathrm{V}$ Leiden accounted for eight of the 11 cases of inferior vena cava thrombosis. Histological examination, performed in 12 patients with factor $\mathrm{V}$ Leiden mutation and in 25 patients without this mutation, showed centrilobular congestion, centrilobular necrosis, as well as fibrosis, in a similar proportion of patients in both groups.

Follow up was similar in patients with and without factor $\mathrm{V}$ Leiden (median 34 months (range 2-432) v 65 months (0.5-198), respectively). All patients were given anticoagulation with fractionated heparin followed by oral vita$\min \mathrm{K}$ antagonists. Two patients with factor $\mathrm{V}$ Leiden developed hepatocellular carcinoma and one was successfully transplanted. Both had indolent inferior vena cava thrombosis. Otherwise, outcomes did not differ significantly in patients with and without factor $\mathrm{V}$ Leiden in terms of thrombolytic therapy (one $v$ six), surgical portosystemic shunting (seven $v$ 19), listing for liver transplantation (two $v$ six), transplantation actually performed (two $v$ two), death (four $v$ six), and death in transplanted patients (one $v$ two). At the end of follow up, the presence of ascites, need for diuretic therapy, serum ALT activity, serum bilirubin, serum albumin, and coagulation factor $\mathrm{V}$ levels did not differ significantly in

Table 3 Prothrombotic states, alanine aminotransferase (ALT) activity, and inferior vena cava (IVC) thrombosis in patients with factor $V$ Leiden

\begin{tabular}{lll}
\hline $\begin{array}{l}\text { Patient } \\
\text { No }\end{array}$ & Prothrombotic state & IVC \\
thrombosis
\end{tabular}

*Times the upper limit of normal values. 
patients with and without factor $\mathrm{V}$ Leiden (data not shown).

\section{Discussion}

In a population of apparently healthy subjects from various areas, the reported prevalence of factor $\mathrm{V}$ Leiden was $0.45-7 \%$, with a mean incidence of 5\% in Europe. ${ }^{26}$ Factor V Leiden has been identified in $20-25 \%$ of patients with venous thromboembolism which makes this anomaly the most common prothrombotic state currently identified. Similarly, the present study has shown that factor $\mathrm{V}$ Leiden was present in $31 \%$ of patients with Budd-Chiari syndrome (95\% CI $20-43)$, a value similar to that found in a recent UK survey. ${ }^{16}$ Thus in patients with Budd-Chiari syndrome, factor $\mathrm{V}$ Leiden is the second most commonly identified prothrombotic state after primary myeloproliferative disorders.

In the present cohort, the first noticeable feature in patients with factor V Leiden was the frequent combination with other prothrombotic states or thrombotic risk factors $(70 \%$ of cases) although other prothrombotic disorders or thrombotic risk factors where found to be equally common in patients with $(24.3 \%)$ and without $(18.4 \%)$ factor $V$ Leiden. A combination of factor V Leiden and other prothrombotic states was documented in three of five cases previously reported by other investigators. ${ }^{11-15}$ These findings indicate that factor V Leiden mutation alone may not be sufficient to cause hepatic vein or inferior vena cava thrombosis. The mutation could act to exacerbate the thrombogenic potential of the other factors. In practical terms, there is a need to investigate other prothrombotic disorders when factor $\mathrm{V}$ Leiden is recognised in a patient with Budd-Chiari syndrome.

The second noticeable feature in patients with factor $\mathrm{V}$ Leiden was presentation with either surprisingly normal or markedly increased serum alanine aminotransferase activity. ALT levels exceeding >50 times the upper limit of normal values in association with decreased coagulation factors indicate massive ischaemic injury. Massive ischaemic injury was almost exclusively encountered in carriers of the factor V Leiden mutation (fig 1). It is noteworthy that in all four patients with massive ischaemic injury and factor V Leiden mutation, an unusual acute condition appeared to trigger acute Budd-Chiari syndrome (table 3, patient Nos 17-20). These observations suggest that resistance to activated protein $\mathrm{C}$ leads to uncontrolled activation of coagulation under particular circumstances. Therefore, factor $\mathrm{V}$ Leiden should be strongly suspected in cases of Budd-Chiari syndrome associated with massive ischaemic injury. This conclusion could be particularly relevant to pregnant or recently delivered woman, as well as to the oral contraceptive user. Indeed, in the present study, among the four patients with massive ischaemic injury, three were pregnant and one was using oral contraceptives. A similar case of uncommon, hyperacute, pregnancy related Budd-Chiari syndrome with factor V Leiden has recently been reported. ${ }^{15}$ Factor V Leiden is known to be strongly associated with other types of venous thromboses occurring during pregnancy and with oral contraceptive use. ${ }^{27-31}$ The reason why the hepatic veins are particularly involved in pregnant women is not clear. We have suggested that trauma to the hepatic veins or terminal inferior vena cava may occur during delivery when abdominal strain and deep respiratory movements compress the veins against the diaphragm. ${ }^{32}$ Contrasting with the highly different presentations of patients with and without factor V Leiden, there were no major differences in their respective outcomes. Thus the impact of factor V Leiden appears to be limited to the initiation of the thrombotic process, at least in patients later given anticoagulation. This observation is consistent with the prothrombotic mechanism induced by factor V Leiden: limitation in the control of ongoing activation of coagulation rather than a trigger for coagulation activation.

The third characteristic found in patients with Budd-Chiari syndrome and factor V Leiden was the frequent association with obstruction of the inferior vena cava, be it membraneous or not. Eight of 11 patients with inferior vena cava thrombosis $(73 \%)$ were carriers of the factor V Leiden mutation whereas only 12 of 52 patients without inferior vena cava thrombosis $(23 \%)$ were carriers $(\mathrm{p}<0.02)$. Previous reports suggested that factor $\mathrm{V}$ Leiden may be less commonly associated with portal vein thrombosis than with hepatic vein thrombosis. ${ }^{16}{ }^{26}$ Taken together, these findings indicate that the type of underlying prothrombotic state may influence the site of thrombosis in the deep veins: little impact on portal vein; impact on hepatic veins that is similar to that on the veins of the lower limbs; and major impact on inferior vena cava. It is tempting to speculate that, for as yet unknown reasons, activation of coagulation takes place specifically at the level of the terminal portion of the inferior vena cava and that this process is not well controlled when factor V Leiden is combined with another prothrombotic state. This local process, when isolated, appears to be indolent and to induce clinically silent obstruction of the inferior vena cava without causing acute ischaemic injury in most patients.

We conclude that factor $\mathrm{V}$ Leiden mutation is likely to be present in almost one third of patients with Budd-Chiari syndrome, usually in association with another prothrombotic state. Factor V Leiden alone appears to be an intrinsically weak thrombophilic factor and to exert its thrombogenic effect mainly when combined with other prothrombotic factors. Factor V Leiden plays a major role in the massive acute forms of the disease, particularly during pregnancy. Moreover, there may be a site specific association with thrombosis of the terminal portion of the inferior vena cava.

\footnotetext{
1 Parker RGF. Occlusion of the hepatic veins in man. Medecine 1959;38:369-402.

2 Dilawari JB, Bambery P, Chawla Y, et al. Hepatic outflow obstruction (Budd-Chiari syndrome): experience with 177 patients and a review of the literature. Medecine 1994;73: $21-36$.
} 
3 Hadengue A, Poliquin M, Vilgrain V, et al. The changing scene of hepatic vein thrombosis: recognition of asymptomatic cases. Gastroenterology 1994;106:1042-7.

4 Bourlière $M$, Le Treut YP, Arnoux D, et al. Acute Budd-Chiari syndrome with hepatic failure and obstruction of the inferior vena cava as the presenting manifestations of hereditary protein C deficiency. Gut 1990;31:949-52.

5 Das M, Caroll SF. Antithrombin III deficiency: an etiology of Budd-Chiari syndrome. Surgery 1985;97:242-5.

6 Valla D, Dhumeaux D, Babany G, et al. Hepatic vein thrombosis in paroxysmal nocturnal hemoglobinuria, a spectrum from asymptomatic occlusion of hepatic venules to fatal Budd-Chiari syndrome. Gastroenterology 1987;93:569-75.

7 Pelletier S, Landi B, Piette JC, et al. Antiphospholipid syndrome as the second cause of non-tumorous BuddChiari syndrome. 7 Hepatol 1994;21:76-80.

8 Valla D, Casadevall N, Lacombe C, et al. Primary myelo-proliferative disorders and hepatic veins thrombosis: a prospective study of erythroid colony formation in vitro in 20 patients with Budd-Chiari syndrome. Ann Intern Med in 20 patients with

9 Valla D, Le MG, Poynard T, et al. Risk of hepatic vein thrombosis in relation to recent use of oral contraceptives: a case-control study Gastroenterology 1986;90:807-11.

10 Dahlbäck B, Hildebrand B. Inherited resistance to actived protein $\mathrm{C}$ is corrected by anticoagulant cofactor activity found to be a property of factor V. Proc Natl Acad Sci USA 1994;91:1396-400.

11 Denninger MH, Beldjord K, Durand F, et al. Budd-Chiari syndrome and factor V Leiden mutation. Lancet 1995;345 525-6.

12 Mahmoud AEA, Wilde JT, Elias E. Budd-Chiari syndrome and factor V Leiden mutation. Lancet 1995;345:526.

13 Mambrini P, Mallet D, O'Callaghan T, et al. Budd-Chiari syndrome and activated protein $\mathrm{C}$ resistance. $\mathcal{F}$ Hepatol 1996;24:246-8.

14 Blashard C, Pasi J, Rolles K, et al. Acute Budd-Chiari syndrome treated by liver transplantation in a woman homozygous for factor V Leiden. Eur 7 Gastoenterol Hepatol 1996;8:925-7.

15 Fickert P, Ramschak H, Kenner L, et al. Acute Budd-Chiari syndrome with fulminant hepatic failure in a pregnant woman with factor V Leiden mutation. Gastroenterology 1996;111:1670-3

16 Mahmoud AEA, Elias E, Beauchamps N, et al. Prevalence of the factor $\mathrm{V}$ Leiden in hepatic and portal vein thrombosis. Gut 1997;40:798-800.

17 Berlin NI. Classification of the polycythemias and initial clinical features in polycythemia vera. In: Wasserman LR, Berk PD, Berlin NI, eds. Polycythemia vera and the myeloproliferative disorders. Philadelphia: WB Saunders, 1995:22-30.

18 Iland $\mathrm{H}$, Laszlo J, Murphy S. Essential thrombocythemia In: Wasserman LR, Berk PD, Berlin NI, eds. Polycythemia vera and the myeloproliferative disorders. Philadelphia: WB Saunders, 1995:293-310.

19 Reid CD, Chanarin I, Lewis J. Formes frustes in myeloproliferative disorders. Identification by the growth of an endogenous erythroid clone in vitro in patients with arterial vascular disease. Lancet 1982;1:14-16.

20 Schubert J, Alvarado M, Uciechowski P, et al. Diagnosis of paroxysmal nocturnal haemoglobinuria using immunophenotyping of peripheral blood cells. Br f Haematol 1991; 79:487.

21 Rosner E, Pauzner R, Lusky A, et al. Detection and quantitative evaluation of lupus circulating anticoagulant activity. Thromb Haemost 1987;57:144.

22 Bertina RM, Koeleman RPC, Koster T, et al. Mutation in blood coagulation factor $\mathrm{V}$ associated with resistance to activated protein C. Nature 1994;369:64-7.

23 Sun X, Evatt B, Griffin JH. Coagulation factor V a abnormality associated with resistance to activated protein C in venous thrombophilia. Blood 1994;83:3120-5.

24 Poort SR, Rosendaal FR, Reitsma PH, et al. A common genetic variation in the 3'-untranslated region of the prothrombin gene is associated with elevated plasma prothrombin levels and an increase in venous thrombosis. Blood 1996;88:3698-703.

25 Denninger MH, Chaït Y, Casadevall N, et al. Cause of portal or hepatic venous thrombosis in adults: the role of multiple concurrent factors. Hepatology 2000;31:587-91.

26 Ridker PM, Miletich JP, Hennekens CH, et al. Ethnic distribution of factor V Leiden in 4047 men and women: implications for venous thromboembolism screening. $7 A M A$ cations for venous

27 Bertina RM, Koeleman BPC, Koster T, et al. Mutation in blood coagulation factor $\mathrm{V}$ associated with resistance to activated protein C. Nature 1994;369:64-7.

28 Vandenbroucke JP, Koster T, Briët E, et al. Increased risk of venous thrombosis in oral-contraceptive users who are carriers of factor V Leiden mutation. Lancet 1994;344:1453-7.

29 Hellgren M, Svensson PJ, Dahlbäck B. Resistance to activated protein $\mathrm{C}$ as a basis for venous thromboembolism associated with pregnancy and oral contraceptives. Am ₹ Obstet Gynecol 1995;173:210-13.

30 Bloemenkamp KWM, Rosendaal FR, Helmerhorst FM, et al. Enhancement by factor $\mathrm{V}$ Leiden mutation of risk of deep-vein thrombosis associated with oral contraceptives containing a third-generation progestagen. Lancet 1995; 346:1593-6.

31 Simioni P, Prandoni P, Lensing AWA, et al. The risk of recurrent venous thromboembolism in patients with an Arg506 $\rightarrow$ Gln mutation in the gene for factor V (factor V Leiden). N Engl f Med 1997;336:399-403.

32 Valla D, Hadengue A, El Younsi M, et al. Hepatic venous outflow block caused by short-length hepatic vein stenoses. Hepatology 1997;23:814-19. 